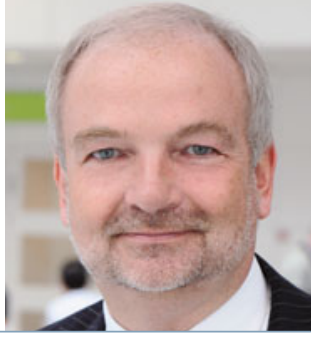

Ralf Birkelbach

\title{
Open Access - Warum brauchen wir eigentlich noch Verlage?
}

Wissenschaft und Kommunikation haben seit jeher eine enge Verbindung. Denn was nützen die neuesten Forschungserkenntnisse, wenn sie die relevante Fachöffentlichkeit nicht erreichen? Umso beachtenswerter sind die Entwicklungen, die sich derzeit im Verlagswesen unter dem Stichwort der Medienkonversion ereignen. Renommierte Publikationen wie die Brockhaus-Enzyklopädie werden nicht mehr fortgesetzt und viele Zeitschriften sehen ihr Geschäftsmodell mit sinkenden Vertriebsauflagen und Anzeigenumsätzen gefährdet. Einzig die Wissenschaftsverlage scheinen es momentan zu schaffen, ihre Kunden von der digitalen Nutzung ihrer Journals und Bücher überzeugen zu können. So macht der Springer-Verlag (u. a. Herausgeber von BIOspektrum) mittlerweile mehr als die Hälfte seiner Umsätze mit seiner Online-Datenbank SpringerLink. Die Vorteile des digitalen Publizierens und Lesens liegen im globalen Science-Geschäft auf der Hand: die Publikation wissenschaftlicher Beiträge verkürzt sich durch online-firstOptionen, leserseitig ist ein Zugriff auf die Inhalte bei entsprechendem 24/7-Datenbankzugriff von jedem Platz der Welt aus möglich. Mithilfe moderner Suchtechnologien können Inhalte vollständig in Sekunden auf relevante Quellen durchsucht werden. Und schließlich können Bibliotheken mit weniger finanziellem Mitteleinsatz der Scientific Community wesentlich mehr Inhalt zur Verfügung stellen.

Bei der rasanten Medienkonversion stellt sich die Frage, ob es zukünftig noch der Wissenschaftsverlage bedarf, oder ob nicht die Forscher selber die Verbreitung ihrer Inhalte mit den Mitteln modernster Informationstechnologien in die Hand nehmen können. Bei der Beantwortung hilft ein Blick auf die klassischen Aufgaben, die von den Verlagen bisher übernommen wurden - und wie die sich im Spiegelbild der Digitalisierung verändern. Da ist zunächst einmal die Selektions- und Qualitätssicherungsfunktion durch die Verlage, d. h. sie treffen eine Vorauswahl der Inhalte, die dann von den Wissenschaftlern im Rahmen des peer review-Prozesses selber begutachtet werden. Hier sind an die Stelle des umständlichen Hantierens und Versendens der Korrekturproofs hohe Entwicklungskosten für entsprechende IT-Lösungen getreten, die den Autoren den peer review-Prozess vereinfachen sollen. Vor allem aber legitimieren die Verlage ihre Daseinsberechtigung im Wissenschaftsbetrieb durch ihre Finanzierungsfunktion, schließlich kommt der Begriff „Verlag“ von „Vorlegen“ d. h. Vorfinanzieren des früher kostspieligen Druck-und Verbreitungsprozesses. An dieser Kernfunktion ändert sich in der digitalen Welt ebenfalls wenig. Sinkenden und vorzufinanzierenden Druck- und Versandkosten stehen heute immense Investitionen für die IT-Entwicklung, die Datenaufbereitung (Metadaten, XML etc.) und den laufenden Betrieb und die Weiterentwicklung von Datenbanken gegenüber.

Bleibt schließlich die Verbreitungs- und Vertriebsfunktion zu prüfen. Auch hier entfällt die Rolle der Verlage nicht, sondern sie modernisiert sich, denn auch digitale Produkte müssen verkauft werden und die Auslieferung und Einspeisung von Daten in unterschiedlichste Bibliotheksserver ist mit hohen Kosten verbunden. Könnte aber nicht auch der Vertrieb der Inhalte durch die Verlage entfallen, wenn die Journals zukünftig - gesponsert durch die forschungsfinanzierenden Institutionen über Open Access-Modelle - frei im Netz distribuiert würden? Die Wissenschaftsverlage müssten dazu ihre teils kritische Haltung gegenüber Open Access als mögliche Existenzbedrohung aufgeben. Der Springer-Verlag hat hier sehr früh mit den Akquisition des Open AccessZeitschriftenverlags BioMedCentral einen Grundstein gelegt, den Wissenschaftlern entsprechende Publikationsmodelle optional neben der Veröffentlichung in Subskriptionszeitschriften anzubieten und ist heute der größte Open Access Anbieter in der Welt.

BIOspektrum steht bei der Digitalisierung vor besonderen Herausforderungen. Denn sie transportiert nicht nur wissenschaftlich fundierte Inhalte innerhalb der Scientific Community, sondern reichert diese mit den ver- schiedensten Nachrichten und Serviceinformationen für Fachgesellschaften, Universitäten und Unternehmen an. Diese inhaltliche Anreicherungsfunktion ist in den klassischen Wissenschaftsjournals und dafür ausgerichteten Onlineformaten und -systemen nicht vorgesehen. Die Zusatzinhalte sind aber genauso wertvoll für die Community der Biowissenschaften wie die selektive Einspeisung von Anzeigen, die einen niedrigen Abonnementpreis insbesondere auch für Gesellschaftsmitglieder sicherstellen sollen. Vor diesem Hintergrund arbeitet der Verlag an innovativen Konzepten, BIOspektrum zukünftig nicht nur in Print, sondern auch in einer Digitalausgabe zur Ausspielung auf den verschiedenen mobilen Endgeräten (Laptops, Tablets wie iPads, Smartphones etc.) zur Verfügung zu stellen. Wie Sie sehen, stellt sich für uns die Frage der „Systemrelevanz" der wissenschaftlichen Verlage nicht. Diese müssen sich aber in einem zunehmend technologiegetriebenen Publikationsgeschäft neu als „Service- und business intelligence-Partner" für Autoren und Leser aufstellen. Wir bei Springer übernehmen hier eine Vorreiterrolle. In diesem Sinne freuen wir uns auf die gemeinsame Arbeit an digitalen Zukunftsprojekten, auch für BIOspektrum!

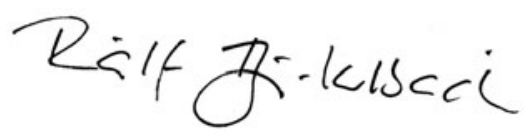

Ralf Birkelbach

Executive Vice President Business \& Technology, Springer Science+Business Media

Korrespondenzadresse:

Dr. Ralf Birkelbach

Springer Science+Business Media

Springer Fachmedien Wiesbaden $\mathrm{GmbH}$ Springer DE

Abraham-Lincoln-Straße 46

D-65189 Wiesbaden

Tel.: $0611-7878-101$

Fax: 0611-7878-78102

Ralf.Birkelbach@springer.com

www.springer.com 